\title{
Verfahren zur Bestimmung des Verschmutzungsgrades von Thermoelementen im Betrieb
}

\author{
Dieter Felkl ${ }^{1}$ und Heinrich Ruser ${ }^{2}$ \\ ${ }^{1}$ Ernst-Abbe-Hochschule Jena, FB Elektrotechnik und Informationstechnik, Jena \\ ${ }^{2}$ Universität der Bundeswehr München, Lehrstuhl für Angewandte Physik und Messtechnik, Neubiberg \\ Kontakt: dieter.felkl@eah-jena.de
}

\section{Einleitung}

Für die Verbesserung der Zuverlässigkeit eines Sensors und die Minimierung der Messabweichungen spielt die Kenntnis des Sensorzustandes eine wichtige Rolle. Dies gilt verstärkt im Zusammenhang mit der Sensorentwicklung für den Einsatz in komplexen industriellen Produktionsanlagen mit einer Vielzahl vernetzter sensorbasierter Teilsysteme (sog. cyber-physischer Systeme) [1]. Kontinuierliche Zustandsüberwachungen der Sensoren sind die Voraussetzung für eine prädiktive Wartung bzw. Instandsetzung.

Ein entscheidender Ansatz zur Zustandsüberwachung besteht in der Ermittlung und Auswertung spezifischer Kennwertänderungen der Sensorelemente, aus denen geeignete Steuersignale generiert werden. Mit selbstüberwachenden Sensoren erfolgt die Zustandsüberwachung adaptiv und automatisch.

Die Temperatur ist in der Prozessmesstechnik und Verfahrenstechnik die am häufigsten gemessene physikalische Größe, so dass der genannte Ansatz anhand der Temperaturmessung mit Berührungsthermometern in fluiden Medien dargestellt und untersucht werden soll.

Lösungsansätze zur Selbstüberwachung bzw. Selbstkalibrierung von Temperatursensoren decken ein weites Spektrum unterschiedlicher physikalischer Prinzipien ab. Der Stand der Technik wird repräsentiert durch die Auswertung:

- implementierter Temperatur-Fixpunktzellen [2] [3] [4],

- der Sprungantwort bei eingespeistem Schleifenstrom [5] [6],

- des thermischen Rauschens eines Widerstands (Rauschthermometrie) [7] [8] [9],

- des elektrischen Widerstandes [10],

- des thermischen Widerstandes [11] bzw. der thermischen Impedanz [12],

- der Temperatur bei sinusförmiger Anregung des Heizelements [13].

Dabei sind Kombinationen mehrerer Prinzipien möglich und üblich [14].

Diese Arbeit liefert einen Beitrag zur automatischen Überwachung des Zustands von Temperatursensoren am Beispiel von Thermoelementen (THE) durch Auswertung des thermischen Widerstandes sowie zur Modellbildung des thermischen Überganges. Ein besonderes Interesse liegt auf der Verifizierung des vorgestellten physikalischen Modellansatzes. Die gezeigte Vorgehensweise ist grundsätzlich auch auf Widerstandsthermometer (RTD) übertragbar.

\section{Methoden und Materialien}

Die Temperaturmessung mit Berührungsthermometern beruht darauf, dass durch die Übertragung thermischer Energie von der Messstelle zum Temperaturfühler mittels konduktiver, konvektiver bzw. radiativer Transportprozesse (Kopplung) die Fühlertemperatur $T_{\mathrm{F}}$ sich der Temperatur $T_{\mathrm{M}}$ des Messobjektes angleicht.

Der thermische Widerstand $R_{\text {th }}$ kann messtechnisch als Quotient der Temperaturdifferenz $T_{\mathrm{FM}}=T_{\mathrm{M}}-T_{\mathrm{F}}$ und der eingespeisten elektrischen Leistung $P$ ermittelt werden:

$$
R_{\mathrm{th}}=\frac{T_{\mathrm{FM}}}{P}
$$

Zusätzlich kann es zu An- bzw. Ablagerungen von im Fluid enthaltenen Partikeln auf dem Thermofühler und ggf. anschließender Sedimentation kommen.

Der thermische Widerstand $R_{\mathrm{th}}(\mathrm{d} r)$ eines durch Ablagerungen, charakterisiert durch den Radiuszuwachs $\mathrm{d} r>0$, verschmutzten Thermofühlers in Luft wird als Reihenschaltung eines konduktiven und konvektiven thermischen Widerstands $R_{\text {thcd }}(\mathrm{d} r)$ und $R_{\text {thcv }}(\mathrm{d} r)$ modelliert [15]:

$$
R_{\text {th }}(\mathrm{d} r)=R_{\text {thcd }}(\mathrm{d} r)+R_{\text {thcv }}(\mathrm{d} r)
$$

Für typische zylindrische Geometrien ergibt sich das folgende Struktur-Modell [16].

$$
R_{\mathrm{th}}(\mathrm{d} r)=\frac{1}{2 \pi \lambda(\vartheta) L} \ln \frac{r_{0}+\mathrm{d} r}{r_{0}}+\frac{1}{2 \pi h(\vartheta) L\left(r_{0}+\mathrm{d} r\right)}
$$

mit den temperaturabhängigen integralen Materialkoeffizienten spezifische thermische Leitfähigkeit $\lambda(\vartheta)$ und thermische Übergangsfunktion $h(\vartheta)$, dem Außenradius $r_{0}$ des unverschmutzten Sensorelements sowie der am thermischen Austausch beteiligten Länge $L$ des Fühlers.

Bei Temperaturmessungen in fluiden Medien dominiert bei kleinen Radiuszuwächsen die konvektive Übertragung des der eingespeisten elektrischen Leistung $P$ äquivalenten Wärmestromes von der Wärmequelle zum Temperaturfühler. In strömenden Medien wird deshalb unter der Bedingung $R_{\text {thcv }} \gg R_{\text {thcd }}$ folgender hyperbolische Modellansatz zur Beschreibung gewählt:

$$
R_{\mathrm{th}}(\mathrm{d} r)=R_{\mathrm{th} 0}+\frac{\Delta R_{\mathrm{th}}}{1+\frac{\mathrm{d} r}{r_{0}}}
$$

Darin steht $R_{\text {tho }}$ für den unteren Grenzwert des thermischen Widerstands $R_{\mathrm{th}}(\mathrm{d} r)$ für $\mathrm{d} r \rightarrow \infty$ und $\Delta R_{\mathrm{th}}$ für seinen Änderungsbereich gegenüber $R_{\mathrm{th}}(\mathrm{d} r=0)$, siehe Abb. 1. 


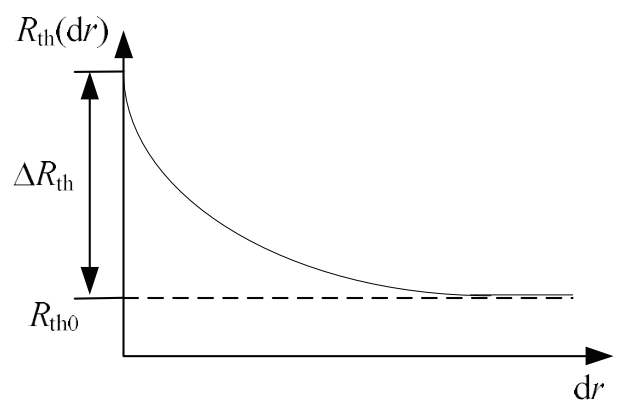

Abb. 1: Schematisierter Graph und Parameter der hyperbolischen Modellfunktion $R_{\mathrm{th}}(\mathrm{d} r)$

Aufgrund der bei Stromfluss $I$ mittels der Leistung $P(I)$ induzierten Temperaturerhöhung $T_{\mathrm{FM}}(I)$ gehen die Gleichungen (1), (3) und (4) in (5), (6) und (7) über.

$$
\begin{gathered}
R_{\mathrm{th}}(I)=\frac{T_{\mathrm{FM}}(I)}{P(I)} \\
R_{\mathrm{th}}(d r, I)=\frac{1}{2 \pi \lambda(\vartheta, I) L} \ln \frac{r_{0}+\mathrm{d} r}{r_{0}} \\
+\frac{1}{2 \pi h(\vartheta, I) L \cdot\left(r_{0}+\mathrm{d} r\right)} \\
R_{\mathrm{th}}(\mathrm{d} r, I)=R_{\mathrm{th} 0}(I)+\frac{\Delta R_{\mathrm{th}}(I)}{1+\frac{\mathrm{d} r}{r(I)}}
\end{gathered}
$$

Erwartbare Abweichungen im physikalischen Modell (3), wie z.B. durch Änderungen der Strömungsverhältnisse am Sensor oder Wechselwirkungen zwischen Geschwindigkeits- und Temperaturfeld innerhalb der Grenzschicht, werden mittels des Parameters $r(I)$ berücksichtigt.

\section{Modellbildung}

Sensorelemente stellen bekanntermaßen die Schnittstellen zwischen unterschiedlichen Energiedomänen dar. Aus diesem Grund erfolgt die Modellierung der Messkette von der Quelle des Messsignals zum Ausgang des Sensorelementes unter Verwendung eines phänomenologischen
Ansatzes mit der Differenz der entsprechenden Potentialgröße als Antriebsgröße (allgemeine Kraft) und der zeitlichen Ableitung (Änderungsgeschwindigkeit) der entsprechenden quantitativen Größe als Strömungsgröße.

Das THE wird als inverser Vierpol mit symmetrischer Zählpfeilorientierung modelliert, siehe Abb. 2. Der Eingangskreis steht für die thermische, der Ausgangskreis für die elektrische Energiedomäne des Sensorelementes [17]. Die Temperaturdifferenz $T_{\mathrm{FV}}$ aus Fühler- und Vergleichsstellentemperatur $T_{\mathrm{F}}$ bzw. $T_{\mathrm{V}}$ stellt die Antriebsgröße und der Wärme(energie)strom $Q$, repräsentiert durch die elektrische Leistung $P_{\mathrm{el}}$, die Strömungsgröße im Eingangskreis dar. Die elektrische Spannung $U$ zwischen den Klemmen des THE bzw. der elektrische Strom I durch diese Klemmen sind die Antriebs- bzw. die Strömungsgröße im Ausgangskreis des Vierpols. Der Sonderfall $U=U_{0}$ gilt für den Leerlauf $(I=0)$ des THE. Der Eingangskreis stellt eine reale Stromquelle, bestehend aus einer idealen Quelle eines der elektrischen Leistung $P$ äquivalenten Wärmestromes $Q$ und dem thermischen Innenwiderstand $R_{\text {th i }}$ - angeordnet zwischen beiden Verbindungsstellen der Thermoschenkel - der Wärmestrom- bzw. Leistungsquelle $P_{\text {el }}$ dar.

Die elektrische Leistung $P(I)$ (8) beinhaltet die Summe der Leistungsanteile der dominierenden thermoelektrischen Effekte, der Peltier-Leistung $P_{\mathrm{P}}$ und der JouleLeistung $P_{\mathrm{J}}$ [18]. Die Peltier-Leistung $P_{\mathrm{P}}$ ist das Produkt aus dem Peltier-Koeffizienten $\Pi$ und elektrischen Strom $I$, die Joule-Leistung $P_{\mathrm{J}}$ das Produkt aus dem effektiven elektrischen Widerstand $R_{\text {eff }}$ und dem Quadrat des elektrischen Stromes $I$, wobei $R_{\text {eff }}$ der Teil des elektrischen Widerstandes des THE ist, an dem sich die Joulesche Leistung $P_{J}$ hinsichtlich der Temperaturauswirkung manifestiert:

$$
P(I)=\Pi I+R_{\mathrm{eff}} I^{2}
$$

Der Ausgangskreis des Vierpoles wird durch eine reale Spannungsquelle - bestehend aus der idealen Spannungsquelle $U_{0}$ und dem elektrischen Widerstand $R$ als Innenwiderstand $R_{\mathrm{i}}$ des THE - modelliert.

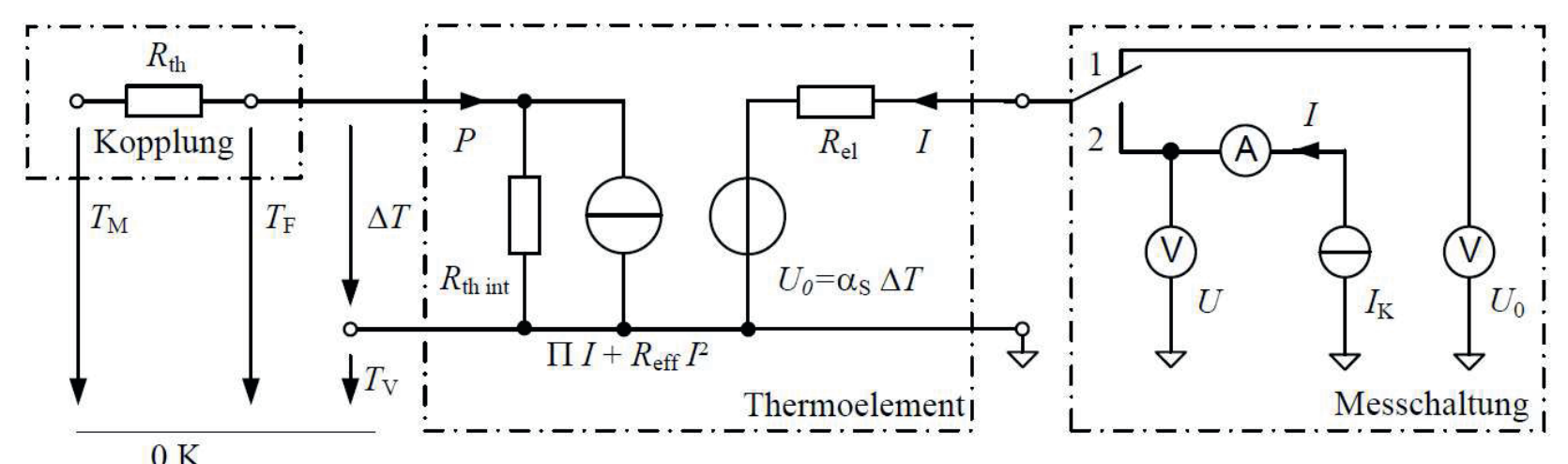

Abb. 2.: Gesamtmodell mit Kopplung an die Messstelle, Thermoelement und Messschaltung 
Die Temperaturabhängigkeit $R(\vartheta)$ des elektrischen Widerstands $R$ wird als lineare Näherung beschrieben:

$$
R(\vartheta)=R_{20}(1+\alpha \Delta \vartheta)
$$

mit $R_{20}=R\left(\vartheta=20^{\circ} \mathrm{C}\right)$, dem linearen Temperaturkoeffizienten $\alpha$ des elektrischen Widerstandes und der gegenüber der Bezugstemperatur $20^{\circ} \mathrm{C}$ auftretenden Temperaturdifferenz $\Delta \vartheta=\vartheta-20^{\circ} \mathrm{C}$.

Die Temperaturdifferenz gegenüber der Umgebung wird extern oder intern aus dem thermischen Widerstand $R_{\text {th }}$ des Sensorelements und der eingetragenen Leistung $P(I)$ (8) generiert.

$$
\Delta \vartheta(I)=R_{\mathrm{th}} P(I)
$$

Aus der quadratischen Abhängigkeit der Leistung $P(I)$ vom Strom $I$ folgt mit der ebenfalls quadratischen Abhängigkeit des elektrischen Widerstandes $R(\vartheta, I)$ von $I$,

$$
R(\vartheta, I)=R_{20}\left(1+\alpha R_{\mathrm{th}} P(I)\right)
$$

sowohl für die elektrischen Leistung $P(I)$ als auch die Temperaturdifferenz $T_{\mathrm{FM}}(I)$ eine Funktion 4. Ordnung von I [11].

\section{Messverfahren und Messbedingungen}

Die Bestimmung des thermischen Widerstandes $R_{\mathrm{th}}(I)$ des Thermofühlers unter Einbeziehung des thermischen Widerstandes der Ankopplung des Sensorelementes an die Messstelle erfolgt durch Anregung des Thermofühlers mit einem konstanten elektrischen Strom $I$, der eine bekannte elektrische Leistung $P(I)$ (8) und damit einen ihr äquivalenten Wärmestrom durch die Fühlerspitze generiert.

Die Fühlertemperatur $T_{\mathrm{F}}(I)$ des THE folgt aus der Temperaturdifferenz $T_{\mathrm{FV}}(I)$ zwischen der Fühlertemperatur $T_{\mathrm{F}}(I)$ und der Vergleichsstellentemperatur $T_{\mathrm{V}}(I) . T_{\mathrm{FV}}(I)$ wird als Quotient aus der im Leerlauf gemessenen Ausgangsspannung $U_{0}$ und dem relativen SeebeckKoeffizienten $\alpha_{\mathrm{S}}$ ermittelt und die Vergleichsstellentemperatur $T_{\mathrm{V}}(I)$ gemessen [18].

$$
\begin{gathered}
T_{\mathrm{F}}(I)=T_{\mathrm{FV}}(I)+T_{\mathrm{V}}(I) \\
T_{\mathrm{FV}}(I)=U_{0} / \alpha_{\mathrm{S}}
\end{gathered}
$$

Die Auswertung der Temperaturdifferenz $T_{\mathrm{FM}}(I)$ (13) über dem thermischen Widerstand $R_{\mathrm{th}}(I)$, beruht auf der Bestimmung der Medientemperatur $T_{\mathrm{M}}$ bei unverschmutztem Fühler durch die Auswertung der Fühlertemperatur $T_{\mathrm{F}}$ im nicht angeregten Zustand (Abb. 2, Messschaltung, Schalterstellung 1) sowie der Auswertung der Fühlertemperatur $T_{\mathrm{F}}(I)$ im angeregten Zustand (Abb. 2, Messschaltung, Schalterstellung 2):

$$
T_{\mathrm{FM}}(I)=T_{\mathrm{F}}(I)+T_{\mathrm{F}}(I=0)
$$

Die Bestimmung des thermischen Widerstandes $R_{\text {th }}$ erfolgt mittels Parameterschätzung gemäß (5) unter Verwendung der Messwerte für $T_{\mathrm{FM}}(I)$ (13) und $P(I)$ (8) sowie dem Modell für $R_{\mathrm{th}}(\mathrm{d} r, I)(6)$.
Auf Grundlage des beschriebenen Verfahrens erfolgt die Charakterisierung eines Typ J Thermoelementes JQIN M15U-150 mit Nickel-Chrom-Mantel in isolierter Ausführung, einem Durchmesser von $2 r_{0}=1,50 \mathrm{~mm}$, einer Länge von $150 \mathrm{~mm}$ sowie mit Standard Anschlussstecker, das im Betrieb von externen Strömungseinflüssen abgeschirmt wird.

Zur Analyse der Modelle (7) bzw. (15) werden Auswertungen mit Anregungsströmen im Bereich 0,2 A $\leq I \leq 1 \mathrm{~A}$ herangezogen. Die Realisierung reproduzierbarer Verschmutzungen erfolgt durch Aufsteckhülsen aus PTFEHülsen mit Außendurchmessern im Bereich $3 \mathrm{~mm} \leq$ $d_{a} \leq 7 \mathrm{~mm}$ von jeweils $50 \mathrm{~mm}$ Länge.

\section{Auswertung und Ergebnisse}

Die Suche nach einem geeigneten Modell für $R_{\mathrm{th}}(\mathrm{d} r, I)$ führt auf das Modell gemäß (7) mit anschließender Schätzung der Parameter $R_{\text {th0 }}, \Delta R_{\text {th }}$ und $r$ für verschiedene Anregungsströme $I$. Dies führt auf das in Abb. 3 dargestellte Kennlinienfeld $R_{\mathrm{th}}(\mathrm{d} r, I)$ mit dem elektrischen Anregungsstrom $I$ als Parameter.

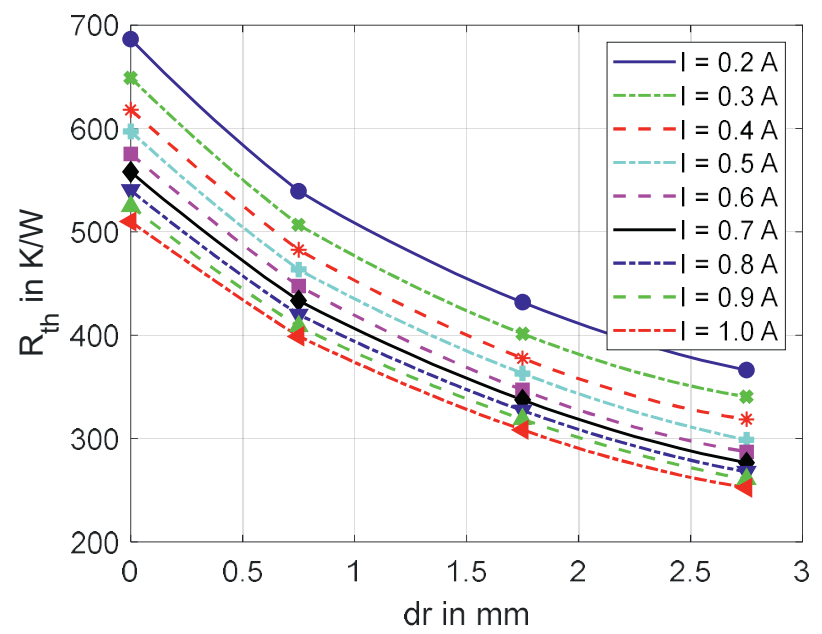

Abb. 3: Modell-Kennlinienfeld $R_{\mathrm{th}}(\mathrm{d} r, I)$ mit den Messwerten für $R_{\mathrm{th}}(\mathrm{d} r, I)$

Die Kennlinien $R_{\mathrm{th}}(\mathrm{d} r, I)$ in Abb. 3 zeigen jeweils für $I=$ const. im unverschmutzten Zustand einen maximalen Startwert $R_{\mathrm{th} 0}+\Delta R_{\mathrm{th}}$ sowie mit zunehmender Verschmutzung $\mathrm{d} r$ die erwartungsgemäß starke hyperbolische Degression des dominanten konvektiven Anteils $R_{\text {thcv }}$ des thermischen Widerstandes.

Mit zunehmendem Strom $I$ und zwangsläufig steigender Temperatur $\vartheta$ sowie größer werdender Materialfunktion $h(\vartheta, I)$ verringert sich gemäß (3) und (6) der dominierende konvektive thermische Widerstand $R_{\text {thcv }}$ weiter und manifestiert sich in einer Verringerung sowohl von $R_{\text {tho }}$ als auch von $\Delta R_{\text {th }}$.

Die relativen Abweichungen zwischen den Modellwerten gemäß (7) und den (Soll-)Messwerten gemäß (6) sind in Abb. 4 dargestellt. Die maximalen relativen Abweichungen sind kleiner als $0,5 \%$. 


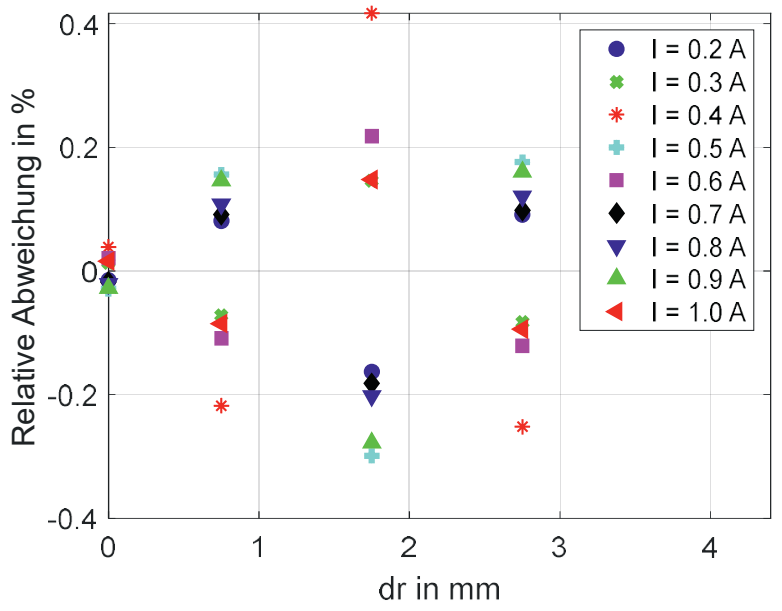

Abb. 4: Relativabweichungen zwischen den Modellwerten gemäß (7) und den Messwerten gemäß (6)

Ziel der weiteren Untersuchungen ist die Darstellung geeigneter Funktionen der Parameter $R_{\text {th } 0}(I), \Delta R_{\text {th }}(I), r(I)$ des hyperbolischen Modells (7). Aus den für verschiedene Anregungsströme $I_{k}$ bestimmten Stützpunkten $R_{\mathrm{th} 0}\left(I_{k}\right)$, $\Delta R_{\text {tho } 0}\left(I_{k}\right)$ und $r\left(I_{k}\right)$ werden die Modellparameter $R_{\mathrm{th} 0}\left(a_{0}, \ldots, a_{m}, I\right), \Delta R_{\mathrm{th}}\left(b_{0}, \ldots, b_{n}, \mathrm{I}\right)$ und $r\left(c_{0}, \ldots, c_{p}, I\right)$ geschätzt. Mit folgendem empirischen Ansatz (14) für die Modellparameter werden maximale relative Abweichungen von den Messwerten (6) von $5 \%$ erzielt, siehe Abb. 5:

$$
\begin{gathered}
R_{\mathrm{th} 0}(I)=\mathrm{a}_{0}+\frac{\mathrm{a}_{1}}{1+\frac{I}{\mathrm{a}_{2}}} \\
\Delta R_{\mathrm{th}}(I)=\mathrm{b}_{0}+\mathrm{b}_{1} I+\mathrm{b}_{2} I^{2}+\mathrm{b}_{3} I^{3}+\mathrm{b}_{4} I^{4} \\
r(I)=\mathrm{c}_{0}+\mathrm{c}_{1} \mathrm{I}
\end{gathered}
$$

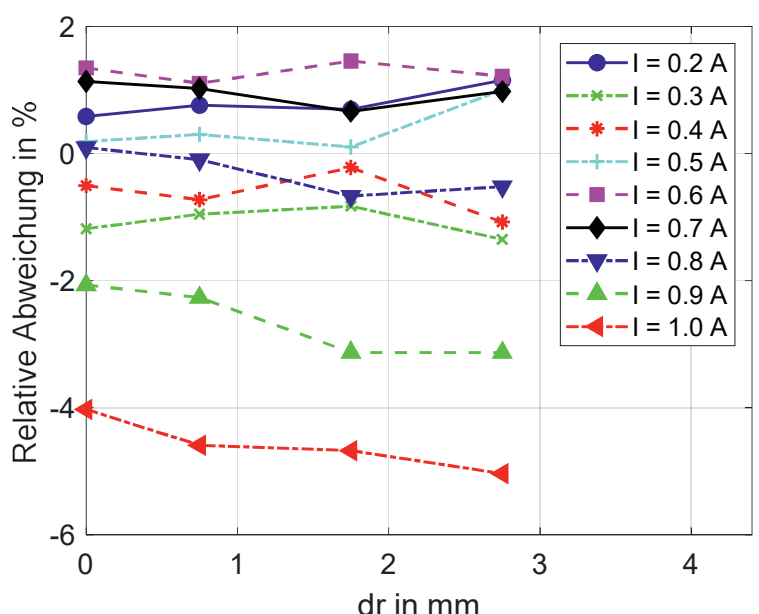

Abb. 5: Relative Abweichungen zwischen den Modellwerten gemäß (14) und den Messwerten gemäß (6).

Entsprechend den dargelegten physikalischen Zusammenhängen hinsichtlich der Abhängigkeiten sowohl der Leistung $P(I)$ als auch der Temperaturdifferenz $T_{\mathrm{FM}}(I)$ vom Strom als Funktionen 4. Ordnung wird in einem zweiten Ansatz auch für den Parameter $R_{\text {tho }}(I)$ ein Polynom 4. Ordnung gewählt. Damit ergeben sich folgende Modellfunktionen:

$$
\begin{array}{r}
R_{\mathrm{th}}(\mathrm{d} r, I)=R_{\mathrm{th} 0}\left(a_{0}, a_{1}, a_{2}, a_{3}, a_{4}, I\right) \\
+\frac{\Delta R_{\mathrm{th}}\left(b_{0}, b_{1}, b_{2}, b_{3}, b_{4}, I\right)}{1+\frac{\mathrm{d} r}{r\left(c_{0}, c_{1}, I\right)}}
\end{array}
$$

mit

$$
\begin{gathered}
R_{\mathrm{th} 0}(I)=a_{0}+a_{1} I+a_{2} I^{2}+a_{3} I^{3}+a_{4} I^{4} \\
\Delta R_{\mathrm{th}}(I)=b_{0}+b_{1} I+b_{2} I^{2}+b_{3} I^{3}+b_{4} I^{4} \\
r(I)=c_{0}+c_{1} I
\end{gathered}
$$

Abb. 6 zeigt die aus den für verschiedene Anregungsströme $I$ bestimmten Modellwerten $R_{\text {tho } 0}(I), \Delta R_{\text {th } 0}(I)$ und $r(I)(7)$ gewonnenen Funktionen $R_{\text {th } 0}\left(\mathrm{a}_{0}, \mathrm{a}_{1}, \mathrm{a}_{2}, \mathrm{a}_{3}, \mathrm{a}_{4}, I\right)$, $\Delta R_{\mathrm{th}}\left(\mathrm{b}_{0}, \mathrm{~b}_{1}, \mathrm{~b}_{2}, \mathrm{~b}_{3}, \mathrm{~b}_{4}, I\right)$ sowie $r\left(\mathrm{c}_{0}, \mathrm{c}_{1}, I\right)$.
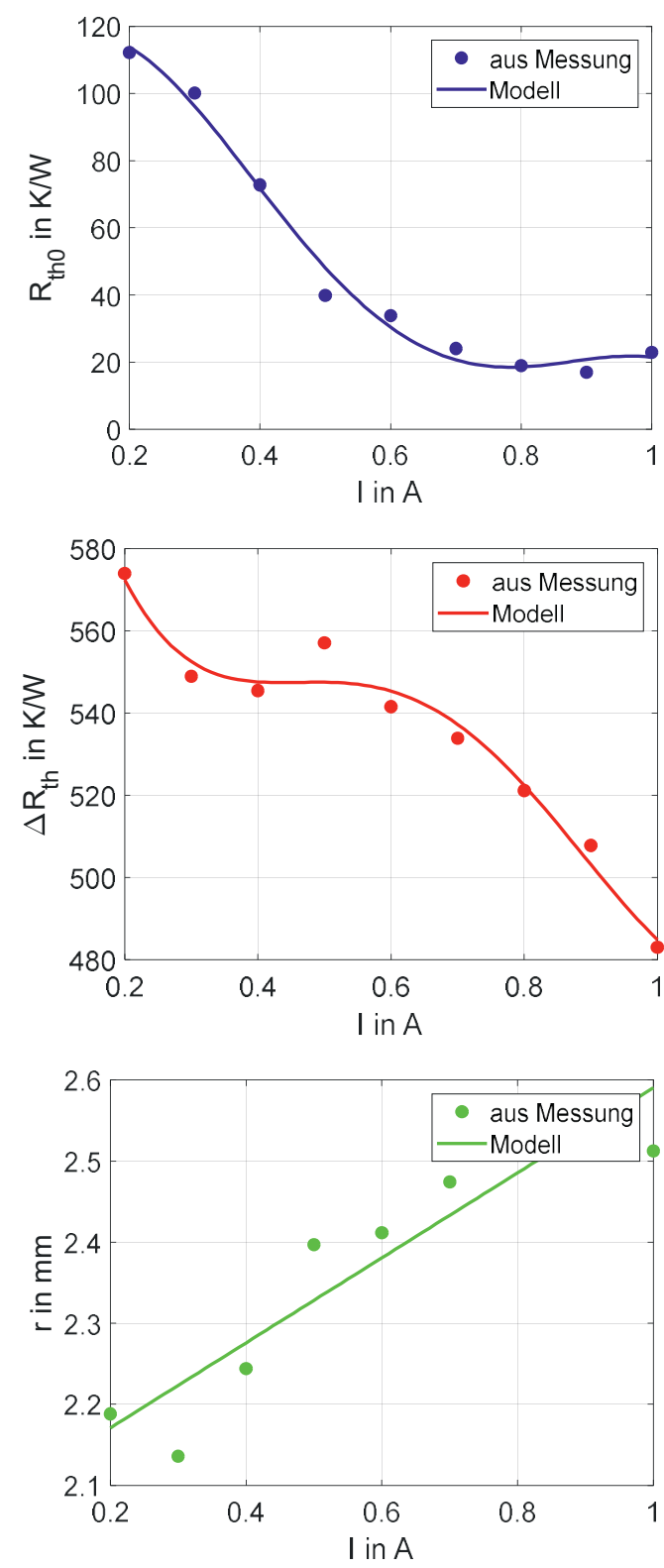

Abb. 6: Mittels hyperbolischem Modellansatz aus Messungen von $R_{\mathrm{th}}(\mathrm{d} r, I)$ bestimmte Parameter $R_{\mathrm{th} 0}(I)$, $\Delta R_{\mathrm{t} 0}(I)$ und $r(I)$ gemäß (16). 
Die relativen Abweichungen $\Delta R_{\mathrm{th}} / R_{\mathrm{th}}$ der Modellwerte gemäß (15) von den Messwerten gemäß (6) sind in Abb. 7 dargestellt.

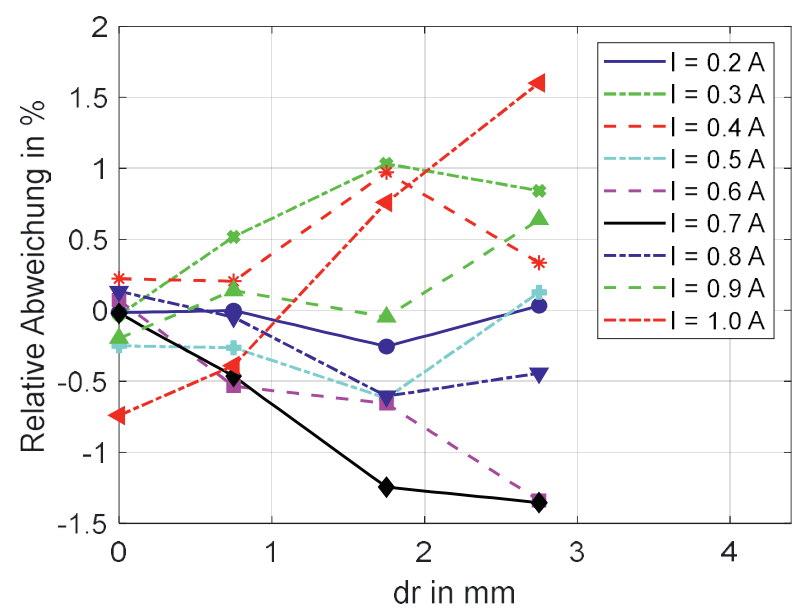

Abb. 7: Relative Abweichungen zwischen den Modellwerten gemäß (15) und den Messwerten gemäß (6)

Die relativen Abweichungen der Modellwerte (15) in Abb. 7 von den Messwerten (6) sind nun kleiner als $1.5 \%$ und plausibilisieren im Vergleich mit den relativen Abweichungen aus Abb. 5 die dargelegten physikalischen Zusammenhänge hinsichtlich der Abhängigkeit der Parameter $R_{\text {th } 0}\left(\mathrm{a}_{0}, \mathrm{a}_{1}, \mathrm{a}_{2}, \mathrm{a}_{3}, \mathrm{a}_{4}, I\right)$ und $\Delta R_{\text {th }}\left(\mathrm{b}_{0}, \mathrm{~b}_{1}, \mathrm{~b}_{2}, \mathrm{~b}_{3}, \mathrm{~b}_{4}, I\right)$ in 4. Ordnung sowie $r(I)$ in 1 . Ordnung von $I$.

\section{Auswertungen zur Selbstüberwachung}

Für die bei der Ankopplung des Messfühlers an die Medientemperatur auftretenden Temperaturen lässt sich formulieren: $T_{\mathrm{M}}=T_{\mathrm{F}}-R_{\mathrm{th}} P$, siehe Abb. 2 .

Mit dem am unverschmutzten Fühler ermittelten thermischen Widerstand $R_{\mathrm{th}}(\mathrm{d} r=0)$ sowie der bei Verschmutzung eintretenden Änderung (Verringerung) des thermischen Widerstandes um $\mathrm{d} R_{\mathrm{th}}(\mathrm{d} r)$

$$
R_{\mathrm{th}}(\mathrm{d} r)=R_{\mathrm{th}}(\mathrm{d} r=0)-\mathrm{d} R_{\mathrm{th}}(\mathrm{d} r)
$$

folgt für die Korrektion $\Delta T_{\mathrm{M}}$ der Medientemperatur $T_{\mathrm{M}}$ :

$$
\begin{aligned}
T_{\mathrm{M}}+\Delta T_{\mathrm{M}} & =T_{\mathrm{F}}-R_{\mathrm{th}}(\mathrm{d} r=0) P+\mathrm{d} R_{\mathrm{th}}(\mathrm{d} r) P \\
\Delta T_{\mathrm{M}} & =\mathrm{d} R_{\mathrm{th}}(\mathrm{d} r) P
\end{aligned}
$$

Die für unterschiedliche Verschmutzungsdicken $\mathrm{d} r$ in Abhängigkeit vom Anregungsstrom $I$ ermittelten Temperaturänderungen $\Delta T_{\mathrm{M}}$ sind in Tab. 1 und $\mathrm{Abb} .8$ bzw. Abb. 9 angegeben.

Tab. 1: Temperaturänderungen $\Delta T_{\mathrm{M}}(\mathrm{d} r, I)$ in $\mathrm{K}$ bei unterschiedlichen Verschmutzungen $d r$.

\begin{tabular}{|c|c|c|c|c|}
\hline & \multicolumn{4}{|l|}{$\mathrm{d} r$ in $\mathrm{mm}$} \\
\hline$\Delta T_{\mathrm{M}}(I)$ in $\mathrm{K}$ & 0 & 0,75 & 1,75 & 2,75 \\
\hline$\Delta T_{\mathrm{M}}(0,2 \mathrm{~A})$ & 0 & 1,4 & 2,5 & 3,3 \\
\hline$\Delta T_{\mathrm{M}}(0,3 \mathrm{~A})$ & 0 & 2,7 & 4,8 & 6,5 \\
\hline$\Delta T_{\mathrm{M}}(0,4 \mathrm{~A})$ & 0 & 4,3 & 7,9 & 10,6 \\
\hline$\Delta T_{\mathrm{M}}(0,5 \mathrm{~A})$ & 0 & 6,4 & 11,6 & 16,1 \\
\hline
\end{tabular}

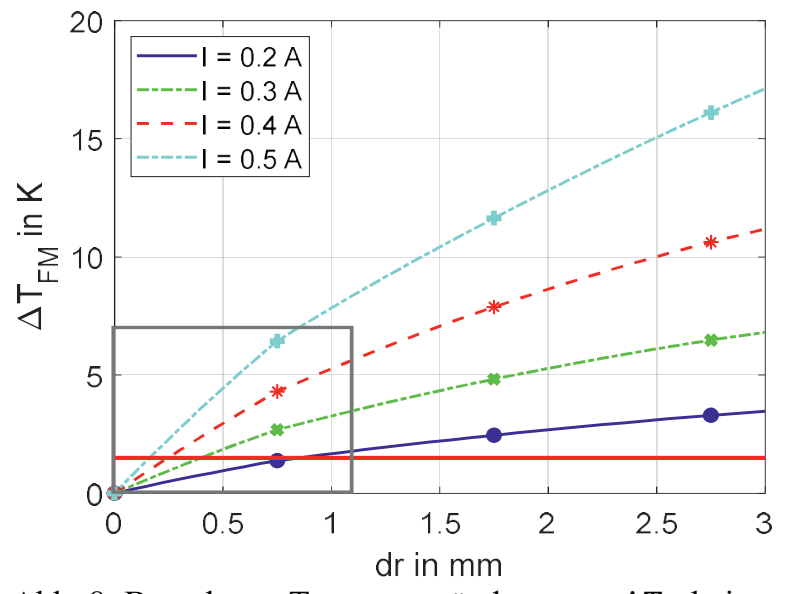

Abb. 8: Berechnete Temperaturänderungen $\Delta T_{\mathrm{M}}$ bei unterschiedlichen Verschmutzungen $\mathrm{d} r$ als Funktion des Anregungsstroms $I$. Der hervorgehobene Ausschnitt ist in Abb. 9 dargestellt.

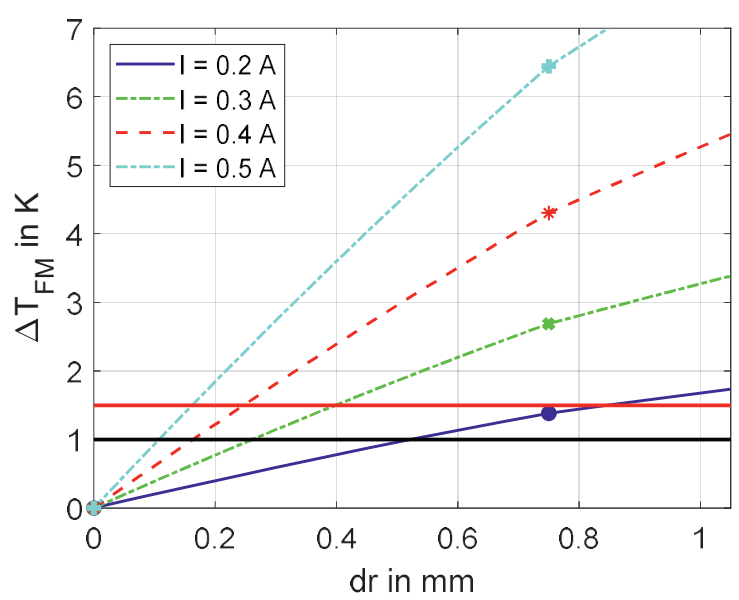

Abb. 9: Ausschnitt aus Abb. 8 zur Verdeutlichung der Temperaturänderung $\Delta T_{\mathrm{M}}$ als Funktion der Verschmutzung $\mathrm{d} r$ bei unterschiedlichen Anregungsströmen $I$ sowie jeweils einer exemplarisch festgelegten Temperaturabweichung $\Delta T_{\mathrm{M} \mathrm{S}}=1,0 \mathrm{~K}$ als Schwellwert bzw. $\Delta T_{\mathrm{M} \max }=1,5 \mathrm{~K}$ als Grenzwert.

Für Temperaturmessfühler werden jeweils Grenzabweichungen - häufig $\Delta T= \pm 1,5 \mathrm{~K}$ [19] - definiert, deren Überschreitung zur Auslösung einer Fehlermeldung führt.

In Tab. 2 werden beispielhaft für vier Anregungsströme $I$ die für eine Grenzwertüberschreitung von $\Delta T_{\mathrm{M} \max }=$ $1,5 \mathrm{~K}$ ermittelten Änderungen des thermischen Widerstandes $\Delta R_{\mathrm{th}}(1,5 \mathrm{~K})$ der durch die Verschmutzung verursachte Radiusänderung $\mathrm{d} r$ zugeordnet.

Tab. 2: Verschmutzungsdicken $\mathrm{d} r$ in $\mathrm{mm}$, die den Änderungen $\mathrm{d} R_{\text {th }}\left(\Delta T_{\mathrm{M}}, \mathrm{d} r\right)$ bei Grenzwert $\Delta T_{\mathrm{M}}=1,5 \mathrm{~K}$ und unterschiedlichen Anregungsströmen $I$ entsprechen.

\begin{tabular}{|c|c|c|c|c|}
\hline$I$ in $\mathrm{A}$ & 0,2 & 0,3 & 0,4 & 0,5 \\
\hline$d R_{t h}(1,5 \mathrm{~K})$ in $\mathrm{K} / \mathrm{W}$ & 130 & 77 & 47 & 28 \\
\hline$d r$ in $\mathrm{mm}$ & 0,8 & 0,4 & 0,25 & 0,15 \\
\hline
\end{tabular}




\section{Zusammenfassung und Ausblick}

Im Beitrag wurde ein neuartiges Verfahren zur automatischen Selbstüberwachung von Berührungstemperatursensoren im Betrieb am Beispiel eines weit verbreitet eingesetzten Thermoelementes vorgestellt. Durch die in aller Regel nicht zu vermeidende mit der Einsatzdauer zunehmende Verschmutzung der Oberfläche des Thermoelements verändert sich die thermische Ankopplung an das Medium und die angezeigte Medientemperatur wird fehlerhaft. Diese graduelle Veränderung lässt sich durch die einhergehende Verminderung des thermischen Widerstands des Sensorelements messtechnisch bestimmen.

Mit Hilfe einer auf physikalischen Gesetzmäßigkeiten beruhenden Modellierung des Verhaltens des thermischen Widerstands in Abhängigkeit vom Verschmutzungsgrad lassen sich aufbauend auf aus Messungen gewonnenen Stützstellen Polynomfunktionen der Modellparameter aufstellen, aus denen der Korrekturterm des Messwertes der Medientemperatur um die verschmutzungsbedingte Temperaturerhöhung automatisch berechnet und zur Selbstkalibration verwendet wird. Der vorgeschlagene physikalische Modellansatz zeigt sehr gute Übereinstimmung mit den Messergebnissen.

Zur Selbstüberwachung des Sensorelements dienen Schwellwerte des gemessenen Temperaturanstiegs, aus denen auf die Verschmutzungsdicke geschlossen werden kann. Bei typischen Grenzwerten von 1,5 K für die quasistationäre Temperaturabweichung lassen sich so bei vergleichsweise kleinem Anregungsstrom von 0,2 A schon Verschmutzungsschichten von $0,8 \mathrm{~mm}$ detektieren. Mit größeren Anregungsströmen steigt die Empfindlichkeit bezüglich der Verschmutzungszunahme überproportional an.

Mit dem dargestellten Verfahren lassen sich über die Bestimmung des Verschmutzungsgrades hinaus weitere praxisrelevante Ursachen von graduellen Messabweichungen im Betrieb detektieren, z.B. zu geringe oder zu starke thermische Kopplung zum Medium durch eine unzureichende Umströmung des Thermoelementes vom Medium oder eine ungenügende Eintauchtiefe in das Medium, mangelnder thermischer Kontakt (Fläche, Kontaktspalt) bei Oberflächenmessungen oder Abrasion bzw. Korrosion von Fühler oder Schutzrohr (siehe VDE 2650-5).

Darüber hinaus ist das für Thermoelemente vorgestellte Verfahren grundsätzlich auch auf andere thermoresistive Sensorelemente wie z.B. Thermistoren, Pt-100 oder spreading resistance Messfühler anwendbar.

\section{Referenzen}

[1] PISTORIUS, J.: Industrie 4.0 - Schlüsseltechnologien für die Produktion, Berlin: Springer Vieweg, 2020.

[2] LEHMANN, H., BERNHHARD F.: Selbstkalibrierende Thermoelemente - Modellierung und Design, Technisches Messen, S. 91-99, 31997.

[3] BOGUHN D.: Miniatur-Fixpunktzellen als Basis selbstkalibrierender elektrischer Berührungsthermometer, Dissertation TU IImenau, 2002.
[4] BOGUHN, D., BUCHNER, R., SCHALLES M., SEEFELD, P.: Verfahren und Vorrichtung zur in situ Kalibrierung eines Thermometers. Patent DE 102010040039, 01032012.

[5] HASHEMIAN, H. M.: New Technology for Remote Testing of Response Time of Installed Thermocouples: Volume I - Background and General Details, Arnold Engineering Development Center, US Air Force (Hrsg.), Arnold Air Force Base, Tennessee, 1992.

[6] YANG, J. C., CLARKE, D. W.: A Self-Validating Thermocouple, IEEE Transactions on Control Systems Technology, Bd. 5, Nr. 2, S. 239-253, 1997.

[7] BRIXY, H.: Rauschthermometer. Patent DT 2347765 C3, 2971976.

[8] BRIXY, H.: Kombinierte Thermoelement-Rauschthermometrie, KFA-Jülich $\mathrm{GmbH}$, Jülich, 1986.

[9] WHITE, D. R., GALLEANO, R., ACTIS A.: The status of Johnson noise thermometry, Metrologica, Nr. 33, S. 325-335, 1996.

[10] FEDOSOV I.I.: Thermocouple Condition Monitoring Using Thermocouple Resistance. Experimental Study, in: Ural Symposium on Biomedical Engineering, Radioelectronics and Information Technology, 2020.

[11] FELKL, D., HORN, M., KARGEL, C.: Selbstüberwachung von Thermoelementen durch Messung des Wärmewiderstandes, Technisches Messen, Bd. 82 (9), S. 463-475, 2015.

[12] HORN, M.: Bestimmung der thermischen Impedanz ohmscher Bauelemente aus periodischen StromSpannungs-Messungen, in: 13. ITG/GMA-Fachtagung Sensoren und Messsysteme, Berlin: VDE, 2006.

[13] KUNTNER, J., KOHL, F., JAKOBY, B.: Simultaneous thermal conductivity and diffusivity sensing in liquids using a micromachined device, Sensors and Actuators A 130-131, S. 62-67, 2006.

[14] ZHOU, K., LI J., WANG W.: A Self-Test, SelfCalibration and Self-Repair Methodology of Thermopile Infrared Detector. Electronics 10, 1167, 2021.

[15] HORN, M., LAZUARDI U., RUSER, H.: Self-controlled PTC sensor for reliable overfill protection of liquids, IEEE Instrumentation and Measurement Technology Conference, Anchorage, AK, USA, 2002.

[16] GRIGULL, U., SANDNER, H.: Wärmeleitung, Berlin, Heidelberg, New York: Springer-Verlag, 1990.

[17] CHAVEZ, J.A., ORTEGA, J. A., SALAZAR, J., TURO A., GARCIA M. J.: SPICE model of thermoelectric elements including thermal effects, IEEE Instrumentation and Measurement Conf., S. 1019-1023, 2000.

[18] SCHMIDT, E.F.: Unkonventionelle Energiewandler, Berlin: Elitera-Verlag, 1975.

[19] DIN EN 60584-1:2014-07 Thermoelemente - Teil 1: Thermospannungen und Grenzabweichungen. 